(NASA-CR-199323) ADDITIONAL

CONSTRAINTS ON CIRCUMSTELLAR OISKS

N96-16907

IN THE TRAPEZIUM CLUSTER

(Harvard-Smithsonian Center for Astrophysics) $13 \mathrm{p}$

Unclas

$63 / 90 \quad 0065138$ 


\title{
ADDITIONAL CONSTRAINTS ON CIRCUMSTELLAR DISKS IN THE TRAPEZIUM CLUSTER ${ }^{1}$
}

\author{
John R. Stauffer, Charles F. Prosser, Lee Hartmann \\ Harvard-Smithsonian Center for Astrophysics, 60 Garden St., Cambridge, MA 02138 \\ Electronic mail: stauffer@cfaharvard.edu, prosser@cfa.harvard.edu, hartmann@cfa.harvard.edu \\ and \\ Mark J. McCaughrean \\ Max-Planck-Institut für Astronomie, Königstuhl 17, 69117 Heidelberg, Germany \\ Electronic mail: mjm@mpia-hd.mpg.de
}

\begin{abstract}
We discuss new constraints on the population of compact ionized sources in the Trapezium Cluster thought to arise from the ionization by the central $O B$ stars of circumstellar disks around low-mass premain sequence stars. We present new HST Planetary Camera observations of two of these candidate disk sources, resolving extended nebulosity around them. One source shows a small-scale ( $\gtrsim 100 \mathrm{AU})$ bow-shock structure, previously seen on larger scales by O'Dell et al. We show that the circumstellar disk model is the most likely one for the majority of sources, although it remains plausible that some of the larger objects could be equilibrium globules. We combine the most complete censuses of compact radio sources and stars in the core region to derive the fraction of the stellar population that may be associated with a circumstellar disk. Our estimate of $25-75 \%$ is comparable to that found for PMS stars in the Taurus-Auriga dark clouds, indicating that the dense cluster environment of the Trapezium has not drastically reduced the frequency of disks seen around pre-main sequence stars.
\end{abstract}

\section{Introduction}

Laques \& Vidal (1979, henceforth LV) discovered several highly ionized compact emission regions close to the Trapezium OB stars in the Orion Nebula using ground-based optical images obtained with an electronographic camera (see also Vidal 1982). Subsequent high-angular resolution radio imaging with the VLA has led to the identification of a large number (presently 49) of compact radio sources throughout the Trapezium Cluster (Garay, Moran, \& Reid 1987 [GMR]; Churchwell et al. 1987 [CFWM]; Felli et al. 1993a; Felli et al. 1993b [FTCCK]). Each LV object is coincident with at least one radio source, most of which have constant thermal spectra and are generally extended (FTCCK), consistent with external ionization. Recently, O'Dell et al. (1993, henceforth OWH) resolved several of the radio sources in HST Wide-Field Camera images taken through narrow-band filters centered on optical emission lines, and discovered a number of new compact but resolved ionized sources without radio counterparts. Also, ground-based adaptive-optics images in the $\mathrm{H} \alpha$ line has increased the number of radio sources in the core of the Trapezium detected as optical emission line nebulosities (McCullough et al. 1994).

The externally ionized class of radio source comprises roughly one third of the total radio source population, and it is these objects and their optical counterparts that are the subject of the present paper. These sources have been referred to in the literature via a number of acronyms, with a 'zoological' theme originated by Garay

\footnotetext{
${ }^{1}$ Based in pan on observations with the NASAESA Hubble Space Telescope, obtained at the Space Telescope Science Institute, which is operated by the Association of Universities for Research in Astronomy, Inc. under NASA contract NAS5-26555.
} 
(1987): PIGS (Partially Ionized Globules); EIDERS (Externally Ionized Disks in the Environs of Radiation Sources-FTCCK); and 'proplyds' (Proto-Planetary Disks-OWH).

The other two-thirds of the radio sources have variable, non-thermal spectra, are generally unresolved, and are more widely spread throughout the Trapezium Cluster. These sources are probably active young stars with gyro-synchrotron emission arising in large photospheric flares, similar to DoAr 21 and V 410 Tau (cf. Cohen \& Bieging 1986; André, Montmerle, \& Feigelson 1987; Brown 1987; Stine et al. 1988). These sources generally fall into the radio category known as FOXES (Fluctuating Optical and X-ray Emitting Sources). A final category, DEERS (Deeply Embedded Energetic Radio Sources), includes sources such as BN and IRc2, embedded in the molecular cloud OMC-1 behind the Trapezium Cluster. Neither the FOXES nor DEERS are discussed further in this paper.

The basic model for the externally ionized sources (Dyson 1968; LV; Vidal 1982; GMR; CFWM) involves the ionization of the outermost layers of a dense concentration of cold gas by the hot luminous OB star members of the Trapezium Cluster, and the subsequent formation of a bow-shock structure by the interaction of the expanding ionized gas with the stellar winds from the OB stars. The latter point is especially emphasized by the images of $\mathrm{OWH}$, as the long axes of the most elongated nebulosities are oriented towards either $\theta^{1}$ Ori C or $\theta^{2}$ Ori $\mathrm{A}$.

The most interesting question concerning these objects is the nature of the cold gas clouds that are being ionized. LV, Vidal (1982), and GMR interpreted the objects simply as cold dense molecular cloud globules, externally ionized following the model of Dyson (1968). However, CFWM emphasized that stars were clearly visible in at least a few of these compact emission regions, and that therefore the simple globule model was suspect. CFWM suggested an alternate model, in which the source of the dense gas is a circumstellar disk surrounding a young star. This is a plausible suggestion, since the sizes and masses of the disks required to explain the radio data ( $\left.\sim 100 \mathrm{AU}, \sim 0.1 M_{\odot}\right)$ are comparable to the properties inferred for disks around low-mass PMS stars (i.e., T Tauri stars) in other regions using other techniques (Adams, Lada, \& Shu 1987, 1988; Beckwith et al. 1990; Strom et al. 1993; Bertout 1989 and references therein). Further support for this circumstellar disk model came with the HST observations of $\mathrm{OWH}$, who resolved several of the sources into ellipsoidal shapes with one end brighter than the other, and some of them showing a central dark region with a star in the middle. They claimed that 12 of their 18 sources were coincident with stars seen in a $2.2 \mu \mathrm{m}$ image from McCaughrean (1988) as reproduced by Genzel \& Stutzki (1989), thus reinforcing the disk arguments of CFWM.

In this paper we report further, higher-resolution HST images of two of the compact radio sources, showing both raw and deconvolved images. These data originate from a larger-scale stellar photometric survey reported in Prosser et al. (1994), henceforth Paper I. We will discuss the continuum flux measured for the sources, and possible physical processes leading to this emission. We also reconsider the various models for the ionized knots. In a companion paper to the present work (McCaughrean \& Stauffer 1994, henceforth Paper II), we describe the most complete census yet made of stars in the inner core of the Trapezium Cluster, as seen in high-resolution near-infrared images. Stellar counterparts to most of the 49 radio sources have now been identified, strengthening the disk model still further. In the present paper, we combine the results from Papers I and II to derive an estimate for the fraction of stars in the core of the Trapezium Cluster that are associated with circumstellar disks. Our results suggest that this fraction is at least as large as seen in lower density star-forming regions such as the Taurus-Auriga dark clouds.

As many of these objects are seen at several wavelengths and are generally given different names or catalogue numbers, there is room for confusion when discussing the various sources. To help alleviate this problem, full cross-reference listings are given in Paper II. In the present paper, we will refer to the radio sources as VLA 1, VLA 2, etc., following the numbering of FTCCK and Paper II; the optical sources of OWH as HST 1, HST 2, etc.; and the ionized nebulosities of Laques \& Vidal (1979) as LV 1, LV 2, etc. Various stellar designations are also used: the optical stars of Jones \& Walker (1988) are referred to as JW001, JW002, etc.; the optical stars of Paper I as PC001, PC002, etc.; and the near-infrared stars of Paper II as TCC001, TCC002, etc. 


\section{HST Observations and Data Analysis}

During August and September 1991, we obtained deep images of 11 fields in the Trapezium Cluster using the Planetary Camera of the HST. The observations were made through two medium-wide filters, the F547M filter centered near $5500 \AA$ and the F875M filter near $8800 \AA$; we will henceforth refer to these as $V$ and $I$ for convenience. Details of the observations can be found in Paper I. The primary aim was to obtain photometry for low-mass stars in the cluster, and therefore we chose field centers which avoid the very brightest stars in the center of the cluster and consequent problems with detector saturation. For that reason, we do not have an image which includes the region within about 20 arcseconds of the $\theta^{1}$ Ori stars, where most of the $L V$ objects and compact emission regions are located (see Paper I for an illustration of the fields observed). Furthermore, our choice of photometric filters was motivated by the desire to avoid the stronger nebular emission lines, in contrast to the study of OWH, whose Wide-Field Camera images were primarily taken through narrow-band filters centered on nebular emission lines. Thus we are somewhat less sensitive to compact $\mathrm{H}$ II regions than $\mathrm{OWH}$, but our point-source limiting magnitude is deeper without competition from large-scale nebular emission, and our spatial resolution is higher.

We found three stars with circumstellar nebulosity in our images, and we illustrate both the raw and the PSFdeconvolved appearance of two of these here. Both of these stars, JW532 and JW558 (identified as PC160 and PC190 in Paper I, and TCC080 and TCC097 in Paper II) were first catalogued in the ground-based proper-motion survey of the Trapezium Cluster by Jones \& Walker (1988). JW558 is associated with HST 1 of OWH and VLA 1 of FTCCK, while JW532 is associated with VLAE of FTCCK. Jones \& Walker (1988) found JW558 to be a proper-motion member of the cluster, but not JW532. However, based on the optical nebulosity detected in our images and the associated radio emission, it seems likely that JW532 is indeed a bona fide member.

Figure 1 (Plate 1) shows the raw $V$ and $I$ images for each of these sources. A non-nebulous star near JW558 is also shown in order to indicate the appearance of a point source with the aberrated HST optics. The scale of the images is 0.04 arcsec/pixel, $\sim 18 \mathrm{AU}$ at the assumed distance to the cluster of $440 \mathrm{pc}$ (Warren \& Hesser 1977). In Figure 2 (Plate 2), we show the same images after application of the "lucy" deconvolution routine in the STSDAS package. The arrows in Figure 2 indicate the direction of the brightest Trapezium OB star, $\theta^{1}$ Ori C.

We believe that our higher-resolution images better illustrate the point source plus inner 'bow-shock' shape of the emission in JW558 than the images of OWH, while the latter show the large-scale bow-shock structure quite clearly. Our $I$-band image exhibits a distinct ridge of emission offset by about $100 \mathrm{AU}$ from the stellar source and extending nearly 180 degrees around it; this structure is not as apparent at $V$. This structure is similar to that seen in high-resolution images of the corresponding radio source VLA 1 (Felli et al. 1993a). Based on its radio variability and negative spectral index, FTCCK tentatively suggested that VLA 1 is a non-thermal source, with emission probably arising from large flares in the atmosphere of the young star itself. However, this source is well-resolved in both the radio and optical continuum images, as well as in the emission-line images of OWH and the near-infrared images of Paper II. Accordingly, we agree with FTCCK's second suggestion, that this source is more likely to be a hybrid, with a flaring PMS star (JW558) sitting at the center of an ionized disk (VLA 1).

We derived magnitudes and colors for the nebulosity around each star from their aperture growth curves, as compared to those for nearby non-nebulous stars. We found both nebulae to have roughly the same color, namely $(V-I) \simeq 2^{m} \cdot 7$. The JW558 nebulosity has an integrated apparent $V$ magnitude of about $17^{m}$. 3 , while the nebulosity for JW532 is about $0^{m} .5$ fainter. Correcting for the mean reddening towards stars in the Trapezium Cluster of $A_{V}=2^{m} .4$ (Herbig \& Terndrup 1986) and converting to flux units, our estimates for the $5500 \AA$ continuum fluxes for the nebulosities associated with JW558 and JW532 are $4.0 \mathrm{mJy}$ and $2.5 \mathrm{mJy}$ respectively.

It is clear that the extended circumstellar emission detected through the narrow-band filters used by OWH is dominated by nebular emission lines; it is not so clear what emission mechanism contributes most to our broadband images of these sources. The $2 \mathrm{~cm}$ radio fluxes for VLA 1 (JW558) and VLAE (JW532) are approximately 11 and $4 \mathrm{mJy}$ (FTCCK; CFWM; GMR), comparable to the dereddened $V$ fluxes and therefore suggestive of thermal 
free-free emission. However, the observed ( $V-I)$ color of both nebulae is somewhat too red to be accounted for by free-free emission. Similarly, radiation from $\theta^{1}$ Ori $C$ scattered by local dust associated with the circumstellar disks would also be bluer than is observed. Therefore, there must be either additional reddening (probably circumstellar) or some other mechanism that contributes primarily in the F875M filter. Other sources might include hydrogen bound-free emission and Paschen lines, several of which fall in that filter. Assuming a temperature of $T=10^{4} \mathrm{~K}$, an electron density of $\mathrm{N}_{e}=10^{6} \mathrm{~cm}^{-3}$, the emission measure of $\sim 10^{9} \mathrm{pc} \mathrm{cm}^{-3}$ calculated for VLA 1 by CFWM, and adopting Case $\mathrm{B}$ (which may not be appropriate for such dense regions), we find that the hydrogen bound-free emission could be of order $10 \mathrm{mJy}$, i.e., comparable in strength to the predicted free-free emission. Similarly, the Paschen lines might contribute a few mly.

As a comparison, we have also measured the color of the large-scale $H$ il region nebular emission in the general vicinity of JW532 and JW558, and find $(V-I) \sim 1 \mathrm{~m} .9$. This is bluer than the circumstellar nebulosity, but not by a large amount. We suspect therefore that the cause of the redder color of the latter is additional extinction. As discussed in more detail in Paper II, the reddening towards the stars with circumstellar nebulosity and extended radio emission cannot be too large, otherwise no ionizing photons could arrive at the star from $\theta^{1}$ Ori $C$ in the first place, and we would see a larger population of radio sources without stellar counterparts. Indeed, LV used Balmer line ratios to measure an extinction of $A_{V} \sim 1^{m} .5$ towards four of their original sources, less than the reddening of $2^{m} .4$ that we have assumed. Nevertheless, the addition of just a magnitude or two more extinction would reconcile the observed circumstellar nebula colors with free-free emission. However, we do not have sufficient data to make that case unambiguously nor determine the physical mechanism providing most of the circumstellar nebular emission. Flux calibrated, carefully background subtracted spectra would resolve this issue.

\section{Models of the Nebular Regions}

The analysis of CFWM suggested that the compact emission regions have ionized densities $\mathrm{N}_{\mathrm{i}} \gtrsim 10^{6} \mathrm{~cm}^{-3}$. If we require the dense gas to be in pressure equilibrium with this ionized material at a temperature $\sim 10^{4} \mathrm{~K}$, then we can calculate the density of the cold gas, if we make reasonable assumptions about the ambient temperature as follows. First, dense molecular regions in Orion are generally at T 30-200K (Goldsmith 1987); second, applicable models of molecular photo-dissociation regions suggest $\mathrm{T} \sim 10^{2} \mathrm{~K}$ for $A V \sim 3-10$ (Tielens \& Hollenbach 1985); third, the black-body equilibrium temperature at the projected distance of JW558 from $\theta^{1}$ Ori C is $\sim 120 \mathrm{~K}$. Therefore, a temperature of $\mathrm{T} \sim 10^{2} \mathrm{~K}$ seems plausible, yielding a cold gas density of $\mathrm{N}_{\mathrm{c}} \gtrsim 10^{8} \mathrm{~cm}^{-3}$.

If such a minimum density cloud has a radius of $100 \mathrm{AU}$ (i.e., roughly equal to the size seen for many of the compact emission regions, $\sim 0.2$ arcsec), its mass is only $10^{-3} M_{\odot}$. As pointed out by CFWM, the ionization by the hot central $O$ stars is likely to lead to evaporation of material from the cloud, an idea strongly supported by the bow-shock structures observed by OWH, and by the nebular structure we observe in JW558. From their radio fluxes, CFWM estimated a mass loss rate of $\sim 10^{-7} M_{\odot} / y r$. This would evaporate the minimum density cloud too rapidly if these compact ionized knots are to last $10^{6} \mathrm{yr}$, i.e., comparable to the age of the Trapezium Cluster. To last that long at the given mass-loss rate, the mass of neutral gas must therefore be of the order of $\sim 0.1 M_{\odot}$ (CFWM). For a self-gravitating isothermal sphere of a given mass $M$, there is a minimum radius for stability,

$$
R_{\text {crit }}=73\left(\frac{M}{0.1 M_{\odot}}\right)\left(\frac{T}{120 \mathrm{~K}}\right)^{-1} \mathrm{AU}
$$

(Bonnor 1956; Ebert 1957). Thus, it is conceivable that some of the larger objects (i.e., diameters larger than $\sim 150 \mathrm{AU}$ or $0.3 \mathrm{arcsec}$ ) could be stable, self-gravitating clouds, which ultimately might collapse to make very low-mass stars. However, this must be considered an unlikely explanation for most sources, since the free-fall time of $\leq 500 \mathrm{yr}$ for such clouds implies extremely short lifetimes, which are at odds with the large number of such sources visible in the region. 
In addition, an optical or near-infrared central star is visible in virtually all of these objects (Paper II), a fact which also rules out a spherical distribution of the cold dense gas around the star. The column density to the center of the $0.1 \mathrm{M}_{\odot}$ cloud considered above would be $\sim 10^{25} \mathrm{~cm}^{-2}$, and dust extinction $\left(A_{V}>10^{3}\right)$ would render any central star invisible at optical and near-infrared wavelengths. A disk model avoids this problem because a large mass can be contained within a small solid angle, leaving the star visible. A mass of $0.1 M_{\odot}$ is comparable to typical disk masses estimated for T Tauri stars in other regions (Beckwith et al. 1990; Strom et al. 1993). In Paper II, approximate masses are derived for stars associated with radio sources, by comparing their near-infrared fluxes with suitably transformed PMS evolution tracks. The majority of the stars classified as 'disk candidates' have masses in the range $0.1-1.5 M_{\odot}$, i.e., they are low-mass stars like the TTauris. The remaining few are somewhat more massive, $\sim 1.5-2.5 M_{\odot}$, and are probably Herbig Ae/Be star analogs.

It seems unlikely that the ionized region being observed is close to the disk surface itself. If this were the case, one might expect to observe a fairly uniform surface brightness across the face of a flat disk, since the angle subtended by the disk as seen from the ionizing source is quite small, and the difference in distances from the ionizing source across the disk is negligible. This picture is consistent with the $V$ filter reconstruction of JW532, but does not agree with the images of JW558, which show a clear brightening on the side toward $\theta^{1}$ Ori C, offset from the position of the central star. The same morphology was seen by OWH for many of their sources, and as they noted, it is difficult to reconcile such an appearance with a simple flat disk model. Instead, we agree with OWH that these are probably some form of bow-shock structure, with limb-brightening toward the ionizing source.

In this explanation, the HST observations resolve the interaction of outflowing ionized material from the circumstellar disk with the $O B$ star wind, rather than tracing the neutral gas structure directly. However, identifying the resolved nebulae seen by HST and the VLA as true bow-shocks may be misleading. Based on a model of the region developed to explain their observations, McCullough et al. (1994) instead place the expected location of the true bow shock between the wind from $\theta^{1}$ Ori $C$ and the disk outfiow at $\sim 2$ arcseconds from the low-mass star. In their model, the arc-like structures seen by HST are hemispherical ionization fronts which shield the circumstellar disk from photo-ionization, but not photo-dissociation. The location of this ionization front is in part determined by the gravity of the star at the center of the circumstellar disk. In support of their modeling, recent mid-infrared imaging by Hayward, Houck, \& Miles (1994) shows that several of the compact radio sources have associated arces of emission at radii around 2-3 arcsec. These arcs appear to be parabolic in shape, with the apex of the parabola oriented towards $\theta^{1}$ Ori $C$, and may be the real bow-shocks at the interface between the outflowing ionized gas and the $O B$ star wind. Similar-sized ares are also seen around several of the same radio sources in emission line images taken during the verification of the new WF/PC-2 on the repaired HST (O'Dell \& Wen 1994).

Clearly, there is much work still to be done in order to explain the extended structures seen at radio, optical, and infrared wavelengths. At present, we conclude that the most attractive explanation for the source of cold gas in these nebulae is a circumstellar disk around a low-mass pre-main sequence star, and that in many cases, the emission arises from an envelope of ionized gas flowing away from the star and interacting with an $O B$ star stellar wind, rather than directly from the disk. Alternatively, there may be an equilibrium shell of ionized gas above the disk, shielding it from direct photo-ionization, with a true bow-shock further out. Also, the possibility remains that a few of the compact ionized sources are indeed massive equilibrium globules (e.g., radio sources VLAM, VLA O, and VLA W; see Paper II), as they appear to be extended, spherical nebulosities in our high-resolution HST and infrared images, with no trace of stellar counterparts. It would not be surprising if there were still a small number of protostellar globules $\left(M \sim 0.5-1 M_{\odot}, R \sim 500-1000 \mathrm{AU}\right)$ in the region, since our cluster member results (Paper I) and other evidence (see, e.g., Zinnecker, MoCaughrean, \& Wilking 1993) indicate that most of the star formation in the Trapezium Cluster has occurred in the last $\sim 10^{6} \mathrm{yr}$. 


\section{The Fraction of Trapezium Cluster Stars with Disks}

A key question now arises concerning these circumstellar disks: what fraction of the cluster population has a disk? Herbig \& Terndrup (1986) noted an apparent lack of classical T Tauri stars in the Trapezium Cluster, and speculated that this might be due to a lack of circumstellar disks amongst the cluster stars, possibly due to disruption by tidal forces during close encounters with other stars (see also Larson 1990). However, OWH suggested that perhaps half the stars in the cluster might have disks, a rough calculation based on the fraction of sources seen in their emission-line images that could be categorized as 'proplyds', i.e., star.disk candidates. We can re-visit this question in a more rigorous fashion by combining the most complete surveys for compact ionized regions (FTCCK) and stars (Paper I and Paper II), and examining the fraction of stars that are associated with disk-like radio sources. There are two major problems to be addressed. First: which radio sources should be counted as possible disks? Second: what fraction of the stellar population actually lies close to the ionizing OB stars, as opposed to merely projected close to them on the sky?

FTCCK classified their radio sources as thermal or non-thermal based mainly on variability, but also on spectral index. Indications were ambiguous for many sources, and these were tentatively classified as possibly thermal, possibly non-thermal, or completely unknown. As we have discussed, the thermal sources are probably compact $\mathrm{H}$ II regions arising from externally ionized disks around PMS stars. By contrast, the non-thermal sources are probably intrinsic stellar radio emitters. Therefore, in deriving statistics conceming the frequency of disks in the Trapezium Cluster, it is only the thermal objects that interest us. However, it is clear that this identification process is far from simple. For example, as discussed earlier, VLA 1 was classified as a possible non-thermal source, whereas based on the extended radio and optical nebulosity, it is more likely to be a thermal source, perhaps with a non-thermally emitting young star (JW558) at its center. There is even ambiguity for VLA 9, one of the most variable radio sources in the region, and confidently classified as a non-thermal stellar emitter by FTCCK. There is indeed an associated star (JW499, TCC052), yet OWH also find a bright emission line cusp and tail (HST 3) around the star, the signature they associate with ionized circumstellar disks. The high-resolution infrared images of Paper II show TCC 052 to be slightly elongated, and new WF/PC-2 continuum images clearly show it to be binary (O'Dell \& Wen 1994). There is also some evidence for extended ionized emission in the WF/PC-2 emission line images, and therefore VLA 9 may be another hybrid object, with a non-thermal stellar source embedded in an externally ionized disk.

In order to proceed, we shall assume that all sources classified by FTCCK as thermal or possibly thermal are externally ionized, and thus are disk candidates. We shall also include sources classified as possibly non-thermal or unknown that are associated with optical emission line nebulosity as seen by LV, OWH, and/or McCullough et al. (1994). We feel that these selection criteria will lead to a reasonable lower limit estimate of the number of possible disks, as they probably exclude some potential disk candidates, including (a) non-thermal radio sources such as VLA 9 and VLA 13, both of which are associated with emission line nebulae (HST 3 and LV6 respectively); (b) radio sources of unknown type that have no optical emission nebulosity but which may be disks with low surface brightness; and (c) OWH nebulae such as HST 11, which, although it exhibits the 'classic' disk morphology and is associated with a star (TCC083), does not have a radio counterpart.

In the models discussed in this paper, material flowing off the circumstellar disk is ionized by the central $\mathrm{OB}$ stars, making it detectable at radio wavelengths. Beyond some distance from the $O B$ stars, the decrease in ionizing flux will lower the resulting gaseous emission below the present radio detection limits. Thus, in order to make a reasonable estimate of the number of stars with disks, we must count only sources within that critical distance. Examination of the sources identified as thermal by FTCCK indicates that most of these sources are within 30 arcsec projected distance from $\theta^{1}$ Ori $C$, the dominant ionization source. In addition, one of the prime candidate disks (VLA 1, JW558), is $26 \operatorname{arcsec}$ from $\theta^{1}$ Ori C. Thus we will choose a radius of $30 \mathrm{arcsec}$ to begin with.

Finally, we must also apply some selection criteria to the stars. In order to make a direct comparison with 
regions such as Taurus-Auriga, it is the fraction of low-mass stars with circumstellar disks that interests us. However, as the present work is concentrated on the core of the Trapezium Cluster, a number of otherwise very rare $O B$ stars are included. These massive stars and their high space density are not typical of the wider cluster. Therefore, we shall exclude the four known OB stars, namely $\theta^{1}$ Ori A, B, C, and D (Levato \& Abt 1976). One of these stars $\left(\theta^{1}\right.$ Ori $B$ ) is resolved into a double in our near-infrared images (Paper II), with a separation of $\sim 0.8$ arcsec. The companion is red and in fact brighter than $\theta^{1}$ Ori $B$ in images we have at $3.6 \mu \mathrm{m}$. The radio source VLA 15, classified by us as a disk candidate, is associated with the red companion to $\theta^{1}$ Ori B. As it seems unlikely that the companion is also an $O B$ star, we will leave it and the associated radio source in our samples.

Using the selection criteria outlined above, there are 20 radio sources that may be disks within a 30 arcsec radius of $\theta^{1}$ Ori C. Within that same angular distance, 75 stars meeting the stellar selection criteria were identified in the infrared census of Paper II. Thus a zeroth order estimate of the fraction of Trapezium Cluster stars with disks is $20 / 75$, i.e., $\sim 27 \%$.

However, we need to correct this estimate for Trapezium Cluster stars which are actually a long way from $\theta^{1}$ OriC, and which only appear close in projection. Again, we will assume that in order to be ionized and detectable, candidate disk sources must be within a true distance from $\theta^{1}$ Ori $\mathrm{C}$ equivalent to 30 arcsec, i.e., $\sim 13000 \mathrm{AU}$ at the $440 \mathrm{pc}$ to the Trapezium ${ }^{2}$. In Paper II, we derive an empirical correction for this projection effect in order to calculate the stellar density near in the core of the cluster. We used the wider-field infrared imaging data of McCaughrean et al. (1991, and in preparation) to determine the cluster 2D radial profile, core radius, and outer radius. Monte-Carlo simulations were then used to model a cluster with the same parameters, and thus to derive the projection correction, which, for the inner 30 arcsec radius, we find to be $2 \pm 0.5$. A more thorough discussion of this projection correction is given in Paper II.

Therefore, we estimate that about half of the stars projected within $30 \mathrm{arcsec}$ from $\theta^{1}$ OriC are actually outside the critical $13000 \mathrm{AU}$ radial distance, and our corrected estimate of the fraction of stars with disks in the Trapezium Cluster is $54 \%$, i.e., more realistically $\sim 1 / 2$, taking into account the considerable uncertainty in the classification of the radio sources.

Altering the radius of the region under consideration will change this number. By looking at ever smaller regions around $\theta^{1}$ Ori $C$, we are likely to probe fainter into the 'disk luminosity function', i.e., the $O$ star will be close enough to ionize disks which are perhaps less favorably oriented, or embedded in more dust. Using the same radio source and stellar selection criteria as above, and the same factor of two correction for the line-of-sight projection effect, we find that the fraction of stars with disks rises, from roughly $54 \%$ at 30 arcsec radius, to $~ 70 \%$ and $\sim 81 \%$ at 25 and $20 \operatorname{arcsec}$ radius respectively. Within a radius of $15 \operatorname{arcsec}$ from $\theta^{1}$ Ori $C$, there are 25 stars and 13 radio sources which match our selection criteria. After correcting for the projection effect, the suggestion is that perhaps all the lower-mass stars in this region have disks.

A second, less stringent but completely independent estimate of the fraction of Trapezium Cluster stars with disks can be made from the HST data of Paper I. Because the HST images did not cover the immediate vicinity of $\theta^{1}$ Ori $C$, we do not have data for the region where the test is most sensitive. However, the Paper I images do cover the region from 20 to 35 arcseconds from $\theta^{1}$ Ori C fairly well, and we can ask what fraction of stars in that annular region shows optical, circumstellar nebulosity. In this region, there are four stars with detected nebulae (JW532, JW558, PC211 and PC178). In the same region, there are a total of 23 stars detected in the HST images with $V>18^{m}$. We choose this limit because all of the stars with detected nebulae have $V>18^{m}$, and disks of similar surface brightness to those we have found would become undetectable for significantly brighter stars, as the brighter stellar PSF would compete with the nebular emission. Thus the raw fraction of stars with disks is $4 / 23$ or $17 \%$. Again correcting by a factor of two for projection effects, the fraction becomes $34 \%$. This is a lower

\footnotetext{
${ }^{2}$ All else being equal, one would expect the emission measure of the externally ionized sources to fall off with distance $d$ from the ionization source as $d^{-2}$.
} 
limit as the region where we have data is relatively far from $\theta^{1}$ OriC, and so some stars with disks may be missed because their nebular surface brightness is low.

In summary, we estimate that $>25 \%$ of the low-mass stars in the Trapezium Cluster have fairly massive $\left(M \sim 0.1 M_{\odot}\right)$ disks. Our best estimate is that this fraction $\gtrsim 50 \%$, the value found in low-density star forming regions such as the Taurus-Auriga dark clouds (Beckwith et al. 1990; Strom et al. 1993). It may even approach $100 \%$, although clearly there is substantial work to be done in classifying the various radio and optical emission line sources, in order for us to make a better comparison with the stellar population. However, it appears that a very dense cluster environment as seen in the core of the Trapezium, with $\sim 5 \times 10^{4}$ stars per cubic parsec (Paper II), does not drastically affect the evolution of disks, at least for timescales less than $1 \mathrm{Myr}$.

As stated earlier, Herbig \& Terndrup (1986) noted that none of the $\sim 10$ stars for which they obtained spectra showed "pronounced T Tauri emission spectra", i.e., that none of these stars are likely to have disks. Our results are in apparent disagreement, assuming that stars with disks necessarily have strong $\mathrm{H \alpha}$ emission. However, the bright nebular $\mathrm{H}$ II emission present throughout the Orion Nebula makes it extremely difficult to measure stellar H $\alpha$ profiles. We agree with the suggestion made by Herbig \& Terndrup (1986) that it would be useful to obtain high resolution, echelle spectra of a larger sample of Trapezium Cluster stars at a site with good seeing in order to place better constraints on the emission line characteristics of these PMS stars.

\section{Conclusions}

Our HST and infrared observations lend additional support to the suggestion of CWFM and OWH that a large fraction of the low-mass, pre-main sequence stars in the Trapezium Cluster have reasonably massive $\left(\sim 0.1 M_{\odot}\right)$ circumstellar disks, although it remains plausible that a few of the larger objects could be equilibrium globules. Combining our data with the radio data, we estimate the circumstellar disk fraction to be $\gtrsim 50 \%$. Apparently the dense star-forming environment of the Trapezium Cluster has not drastically reduced the frequency of premain sequence disks. Additional optical and infrared imaging and spectroscopy are needed to confirm the disk model and to constrain physical conditions in the dense gas. In addition, infrared excess should be measured using thermal-infrared $(>3 \mu \mathrm{m})$ imaging photometry. Indeed, the latter technique should be used throughout the Trapezium Cluster in order to reveal stars that have disks which are not significantly externally ionized, either because they are too far from the Trapezium $\mathrm{OB}$ stars, or because they are too deeply embedded. Only then will we know if the apparently high frequency of stars with disks seen in the core is typical of the whole cluster. Finally, millimeter interferometry should be used to see if the cold outer regions of the disks have survived the crowded cluster environment.

Looking at the wider perspective, there are compelling reasons to follow-up with more extensive studies of disks around stars in young clusters. There is growing evidence that the majority of stars in the Galaxy are formed in dense stellar clusters and aggregates in GMCs, as opposed to the relative isolation of dark clouds such as Taurus-Auriga (Zinnecker et al. 1993; Lada, Strom, \& Myers 1993). As disks are integral part of modern star formation theories, it is important to understand how such models may have to be modified to account for the very dense environments of clusters, where disks may interact. While the apparently inevitable interactions in the crowded core the Trapezium Cluster seem not to have dramatically affected the fraction of stars with disks, there is the caveat that in the Trapezium we are seeing structures on the order of 200-500 AU in size, and that the larger and colder outer parts of the disks (500-2000 AU) may have been stripped in disk-disk interactions.

Also, if most stars form in clusters with an apparently high frequency of associated disks, then we should address planet formation in a cluster environment. This is an attractive topic in light of two features of the only confirmed planetary system to date, namely our own. The tilt of the ecliptic relative to the solar rotation axis could be explained as resulting from disk-disk interactions in a dense cluster environment (Mottmann 1977; Herbig 1982; Tremaine 1991; Heller 1993). Also, supernova pollution of the protoplanetary disk, as evidenced by anomalies 
found in the metal content of some meteorites, would be a natural consequence of formation near an $O B$ cluster (Reeves 1978).

Support for this work was provided by NASA through grant number GO2595.01-87A from the Space Telescope Science Institute, which is operated by the Association of Universities for Research in Astronomy, Inc., under NASA contract NAS5-26555. JRS also acknowledges support from NASA under the Long-Term Space Astrophysics Grant NAGW-2698.

Copies of this preprint, with its figures, can be obtained over the Internet as follows:

- Via anonymous ftp: connect to spit $f$ ire .mpia - hd . mpg . de, login as $f t p$, with your e-mail address as password. cd to pub/preprints, and get the README file for more information.

- Via the World-Wide-Web: connect to http://spitfire.mpia-hd.mpg.de/Preprints.html and follow the links relevant to this paper. 


\section{REFERENCES}

Adams, F., Lada, C. J., \& Shu F. H. 1987, ApJ, 313, 788

Adams, F., Lada, C. J., \& Shu F. H. 1988, ApJ, 326, 865

Andre, P., Montmerle, T., \& Feigelson, E. D. 1987, AJ, 93, 1182

Beckwith, S. V. W., Sargent, A. I., Chini, R. S., \& Güsten, R. 1990, AJ, 99, 924

Bertout, C. 1989, ARA\&A, 27, 351

Bonnor, W. B. 1956, MNRAS, 116, 351

Brown, A., 1987, ApJ, 322, L31

Churchwell, E. B., Felli, M., Wood, D. O. S., \& Massi, M. 1987, ApJ, 321, 516 (CFWM)

Cohen, M. \& Bieging, J. H. 1986, AJ, 92, 1396

Dyson, J. E. 1968, Astrophys. Space Sci., 1, 388

Ebert, R. 1957, Z Astrophys., 42, 263

Felli, M., Churcbwell, E. B., Wilson, T. L., \& Taylor, G. B. 1993a, A\&AS, 98, 137

Felli, M., Taylor, G. B., Catarzi, M., Churchwell, E. B., \& Kurtz, S. 1993b, A\&A, 101, 127 (FTCCK)

Garay, G., Moran, J. M., \& Reid, M. J. 1987, ApJ, 314, 535 (GMR)

Garay, G. 1987, Rev. Mex. Astron. Astrophys., 14, 489

Genzel, R. \& Stutzki, J. 1989, ARA\&A, 27, 41

Goldsmith, P. F. 1987, in Interstellar Processes, eds. D. J. Hollenbach \& H.A. Thronson, (Dordrecht: D. Reidel), p51

Heller, C. H. 1993, ApJ, 408, 337

Herbig, G. H. 1982, in Symposium on the Orion Nebula to Honor Henry Draper, Ann. N. Y. Acad. Sci., 395, 64

Herbig, G. H. \& Terndrup, D. M. 1986, ApJ, 307, 609

Hayward, T. L., Houck, J. R., \& Miles, J. W. 1994, ApJ, submitted

Jones, B. F. \& Walker, M. F. 1988, AJ, 95, 1755

Lada, E. A., Strom, K. M., \& Myers, P. C. 1993, in Protostars and Planets III, eds. E. H. Levy \& J. I. Lunine, (Tucson: Univ. of Arizona Press), p245

Laques, P. \& Vidal, J.-L. 1979, A\&A, 73, 97

Larson, R. B. 1990, in Physical Processes in Fragmentation and Star Formation, eds. R. Capuzzo-Dolcetta, C. Chiosi, \& A. Fazio, (Dordrecht: Kluwer), p389

Levato, H. \& Abt, H. A. 1976, PASP, 88, 712

MoCaughrean, M. J. 1988, PhD thesis, University of Edinburgh

MoCaughrean, M. J., Zinnecker, H., \& Rayner, J. T. 1991, Mem. S. A. It., 62, 715

McCaughrean, M. J. \& Stauffer, J. R. 1994, AJ, in press (Paper II)

McCullough, P. R., Fugate, R. Q., Ellerbroek, B. L., Higgins, C. H., Spinhirne, J. M., Moroney, J. F., \& Cleis, R. A. 1994, ApJ, submitted

Mottmann, J. 1977, Icarus, 31, 412

O’Dell, C. R., Wen, Z., \& Hu, X. 1993, ApJ, 410, 696 (OWH)

O'Dell, C. R. \& Wen, Z. 1994, ApJ, submitted 
Prosser, C. F., Stauffer, J. R., Hartmann, L., Soderblom, D. R., Jones, B. F., Wemer, M. W., \& McCaughrean, M. J. 1994, ApJ, 421, 517 (Paper I)

Reeves, H. 1978, in Protostars and Planets, ed. T. Gehrels, (Tucson: Univ. of Arizona Press), p399

Stine, P. C., Feigelson, E. D., Andre, P., \& Montmerle, T. 1988, AJ, 96, 1394

Strom, S. E., Edwards, S., \& Skrutskie, M. F. 1993, in Protostars and Planets III, eds. E. H. Levy \& J. I. Lunine, (Tucson: Univ. of Arizona Press), p837

Tielens, A. G. G. M. \& Hollenbach, D. 1985, ApJ, 291, 722

Tremaine, S. 1991, Icarus, 89, 95

Vidal, J.-L. 1982, in Symposium on the Orion Nebula to Honor Henry Draper, Ann. N. Y. Acad. Sci., 395, 176

Warren, W. H. \& Hesser, J. E. 1977, ApJS, 34, 115

Zinnecker, H., McCaughrean, M. J., \& Wilking, B. A. 1993, in Protostars and Planets III, eds. E. H. Levy \& J. I. Lunine, (Tucson: Univ. of Arizona Press), p429 


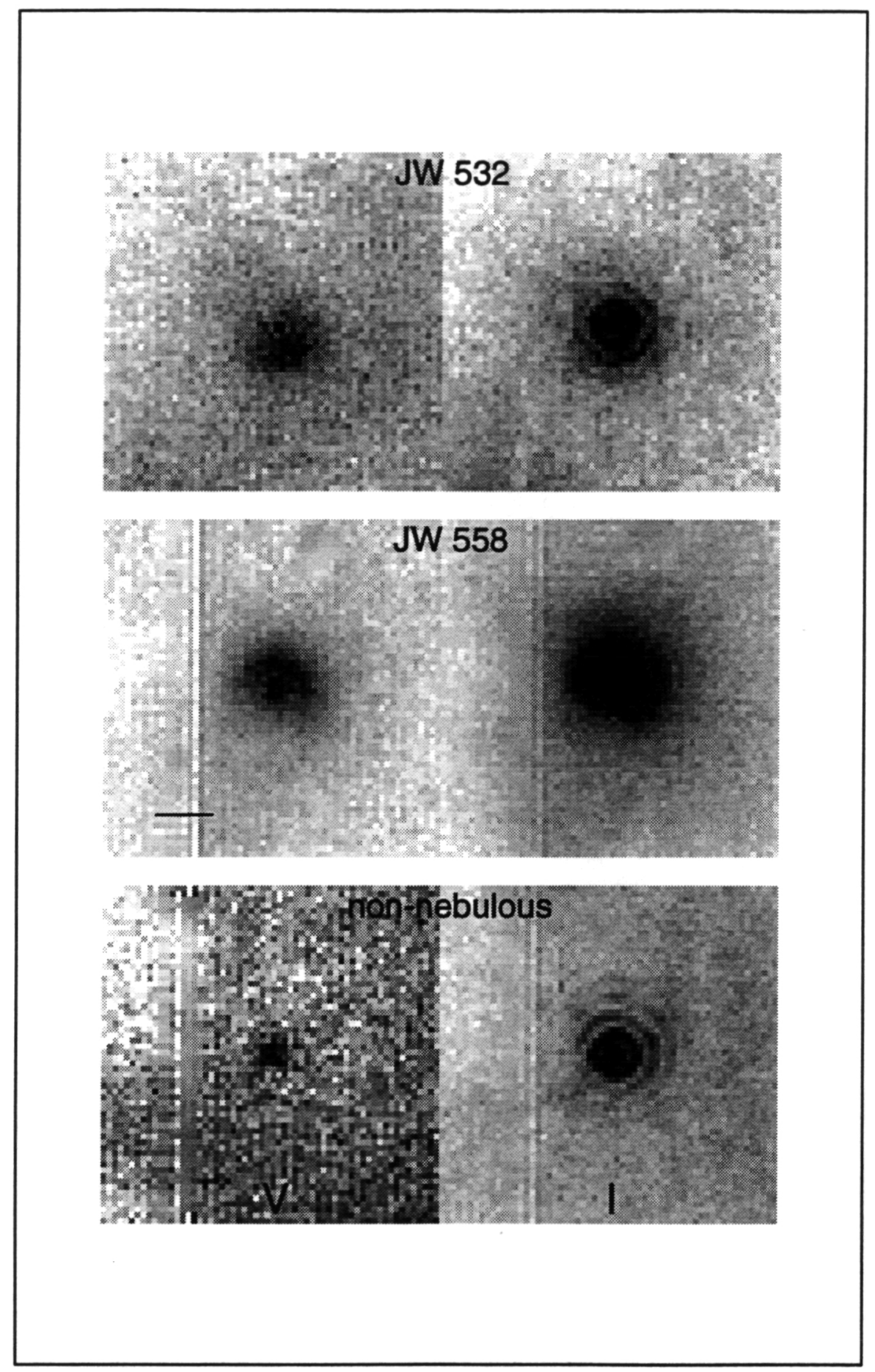

Fig. 1.- Raw Planetary Camera $V$ (F547M) and $I$ (F875M) images of JW532, JW558, and a non-nebulous star near JW558. The latter star indicates the appearance of a point source with the aberrated HST optics. The scale is $0.044 \mathrm{arcsec} /$ pixel and the bar in the $V$ image of JW558 is 0.5 arcsec long. 


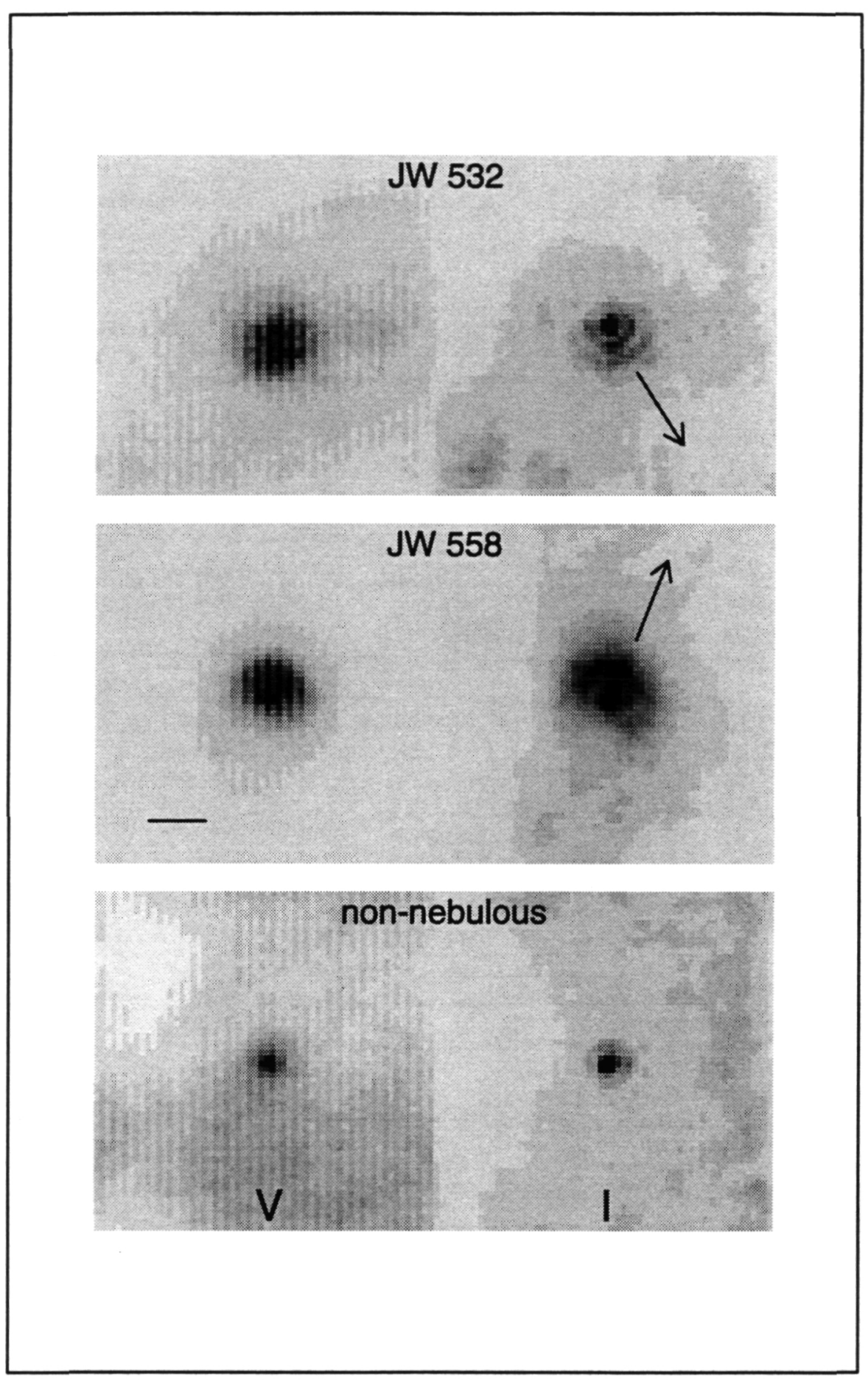

Fig. 2. - The same three stars after "lucy" deconvolution. The arrows indicate the direction towards $\theta^{1}$ Ori $C$. The pronounced striping in the $V$ images is an artifact. 